\title{
Restricted One-dimensional Central Extensions of the Restricted Filiform Lie Algebras $\mathfrak{m}_{0}^{\lambda}(p)$
}

\author{
Tyler J. Evans \\ Department of Mathematics \\ Humboldt State University \\ Arcata, CA 95521 USA \\ evans@humboldt.edu \\ Alice Fialowski* \\ Institute of Mathematics \\ University of Pécs and Eötvös Loránd University \\ Hungary \\ fialowsk@ttk.pte.hu, fialowsk@cs.elte.hu
}

Keywords: restricted Lie algebra; central extension; cohomology; filiform Lie algebra

Mathematics Subject Classification: 17B50, 17B56

\begin{abstract}
Consider the filiform Lie algebra $\mathfrak{m}_{0}$ with nonzero Lie brackets $\left[e_{1}, e_{i}\right]=e_{i+1}$ for $1<i<p$, where the characteristic of the field $\mathbb{F}$ is $p>0$. We show that there is a family $\mathfrak{m}_{0}^{\lambda}(p)$ of restricted Lie algebra structures parameterized by elements $\lambda \in \mathbb{F}^{p}$. We explicitly describe both the ordinary and restricted 1-cohomology spaces and show that for $p \geq 3$ these spaces are equal. We also describe the ordinary and restricted 2-cohomology spaces and interpret our results in the context of one-dimensional central extensions.
\end{abstract}

${ }^{*}$ The final version of the paper was written during the stay of the second author at the Max-Planck Institute for Mathematics Bonn, Germany. 


\section{Introduction}

In the past years a lot of attention has been paid to nilpotent $\mathbb{N}$-graded Lie algebras of maximal class. By $\mathbb{N}$-graded we mean that the Lie algebra is the direct sum of subspaces $\mathfrak{g}_{i}, i \in \mathbb{N}$ such that $\left[\mathfrak{g}_{i}, \mathfrak{g}_{j}\right] \subset \mathfrak{g}_{i+j}$. A Lie algebra of maximal class is a graded Lie algebra

$$
\mathfrak{g}=\bigoplus_{i=1}^{\infty} \mathfrak{g}_{i}
$$

over a field $\mathbb{F}$, where $\operatorname{dim}\left(\mathfrak{g}_{1}\right)=\operatorname{dim}\left(\mathfrak{g}_{2}\right)=1$, $\operatorname{dim}\left(\mathfrak{g}_{i}\right) \leq 1$ for $i \geq 3$ and $\left[\mathfrak{g}_{i}, \mathfrak{g}_{1}\right]=\mathfrak{g}_{i+1}$ for $i \geq 1$. Algebras of maximal class are either finite dimensional, or all their proper factors are finite dimensional. These infinite dimensional algebras can be viewed as (projective) limits of nilpotent Lie algebras of maximal class. They are also called narrow, thin, or coclass 1 Lie algebras.

If the number of generators of such Lie algebra is the minimal 2, then all such Lie algebras are classified in characteristic 0 . In fact, all $\mathbb{N}$-graded infinite dimensional Lie algebras with two generators $e_{1}$ and $e_{2}$ have been classified in [6], and exactly 3 of those are of filiform type. An $n$-dimensional nilpotent Lie algebra is filiform, if $\operatorname{dimg}^{2}=n-2, \ldots, \operatorname{dimg}^{k}=n-k, \ldots$, $\operatorname{dim} \mathfrak{g}^{n}=0$ where $\mathfrak{g}^{k}=\left[\mathfrak{g}, \mathfrak{g}^{k-1}\right], 2 \leq k \leq n$. We also call their projective limit filiform type. We list them with the nontrivial bracket structures:

$$
\begin{aligned}
\mathfrak{m}_{0}: & {\left[e_{1}, e_{i}\right]=e_{i+1}, \quad i \geq 2, \quad i \in \mathbb{N} } \\
\mathfrak{m}_{2}: & {\left[e_{1}, e_{i}\right]=e_{i+1}, \quad i \geq 2, \quad\left[e_{2}, e_{j}\right]=e_{j+2}, \quad j \geq 3, \quad i, j \in \mathbb{N} } \\
\mathfrak{V}: & {\left[e_{i}, e_{j}\right]=(j-i) e_{i+j}, \quad i, j \geq 1 . }
\end{aligned}
$$

In the finite-dimensional case in characteristic zero, the classification of finite-dimensional $\mathbb{N}$-graded filiform Lie algebras is also done in [12]. They include the natural "truncations" of the above three algebras $\mathfrak{m}_{0}(n), \mathfrak{m}_{2}(n)$ and $\mathfrak{V}(n)$, which are obtained by taking the quotient by the ideal generated by $e_{n+1}$, but there are other families as well.

The picture is more complicated in positive characteristic, see [1, 2, 11], but $\mathfrak{m}_{0}, \mathfrak{m}_{2}$ and their truncations always show up.

The cohomology of $\mathbb{N}$-graded Lie algebras of maximal class has been studied extensively over a field of characteristic zero $([6,7,15])$, but for positive characteristic, much less is known. Recently it was shown that over a field of 
characteristic two, the algebras $\mathfrak{m}_{0}(n)$ and $\mathfrak{m}_{2}(n)$ have the same Betti numbers [14, and the cohomology spaces with trivial coefficients are obtained in this case. In fact, these cohomology spaces are isomorphic [13]. For the truncated finite dimensional analogues, the first 3 cohomology spaces are known over $\mathbb{Z}_{2}$.

In this paper we show if the field $\mathbb{F}$ has characteristic $p>0$, the Lie algebra $\mathfrak{m}_{0}(p)$ admits the structure of a restricted Lie algebra. In fact, we show that there is a family $\mathfrak{m}_{0}^{\lambda}(p)$ of such restricted Lie algebra structures parameterized by elements $\lambda \in \mathbb{F}^{p}$. Using the ordinary Chevalley-Eilenberg complex and the partial restricted complex in [5], we calculate the dimensions of both the ordinary cohomology $H^{q}\left(\mathfrak{m}_{0}^{\lambda}(p)\right)$ and the restricted cohomology $H_{*}^{q}\left(\mathfrak{m}_{0}^{\lambda}(p)\right)$ for $q=1,2$, and we explicitly describe bases for these spaces.

The organization of the paper is as follows. Section 2 contains the constructions of the Lie algebras and restricted Lie algebras under consideration including explicit descriptions of the Lie brackets, [p]-operators and both ordinary and restricted cochain spaces and differentials. Section 3 contains the computation of both the ordinary cohomology $H^{1}\left(\mathfrak{m}_{0}^{\lambda}(p)\right)$ and restricted cohomology $H_{*}^{1}\left(\mathfrak{m}_{0}^{\lambda}(p)\right)$, and in particular it is shown that these spaces are equal for primes $p \geq 3$. Section 4 contains the computations of $H^{2}\left(\mathfrak{m}_{0}^{\lambda}(p)\right)$ and $H_{*}^{2}\left(\mathfrak{m}_{0}^{\lambda}(p)\right)$. In Section 5, we interpret our results in the context of onedimensional (both ordinary and restricted) central extensions.

Acknowledgements. The authors are grateful for helpful conversations with Dmitry B. Fuchs, and for useful suggestions from the referee.

\section{Preliminaries}

\subsection{The Lie Algebra $\mathfrak{m}_{0}(p)$ over $\mathbb{F}$}

Let $p>0$ be a prime, and let $\mathbb{F}$ be a field of characteristic $p$. Define the $\mathbb{F}$-vector space

$$
\mathfrak{m}_{0}(p)=\operatorname{span}_{\mathbb{F}}\left(\left\{e_{1}, \ldots, e_{p}\right\}\right),
$$

and define a bracket on $\mathfrak{m}_{0}(p)$ by

$$
\left[e_{1}, e_{i}\right]=e_{i+1}, \quad 1<i<p,
$$

with all other brackets $\left[e_{i}, e_{j}\right]=0$. Note that $\mathfrak{m}_{0}(p)$ is a graded Lie algebra with $k$ th graded component $\left(\mathfrak{m}_{0}(p)\right)_{k}=\mathbb{F} e_{k}$ for $1 \leq k \leq p$. If $\alpha_{i}, \beta_{i} \in \mathbb{F}$ and 


$$
\begin{aligned}
g=\sum_{i=1}^{p} \alpha_{i} e_{i}, h=\sum_{i=1}^{p} \beta_{i} e_{i}, \text { then } & \\
& {[g, h]=\sum_{j=3}^{p}\left(\alpha_{1} \beta_{j-1}-\alpha_{j-1} \beta_{1}\right) e_{j} . }
\end{aligned}
$$

\subsection{The Restricted Lie Algebras $\mathfrak{m}_{0}^{\lambda}(p)$}

For any $j \geq 2$ and $g_{1}, \ldots, g_{j} \in \mathfrak{m}_{0}(p)$, we write the $j$-fold bracket

$$
\left[g_{1}, g_{2}, g_{3}, \ldots, g_{j}\right]=\left[\left[\ldots\left[\left[g_{1}, g_{2}\right], g_{3}\right], \ldots,\right] g_{j}\right] \text {. }
$$

Equation (1) implies that the center of the algebra is $Z\left(\mathfrak{m}_{0}(p)\right)=\mathbb{F} e_{p}$, and that any $p$-fold bracket $\left[g_{1}, g_{2}, g_{3}, \ldots, g_{p}\right]=0$. In particular, equation (1) implies $(\operatorname{ad} g)^{p}=0$ for all $g \in \mathfrak{m}_{0}(p)$. Therefore $\left(\operatorname{ad} e_{k}\right)^{p}=0$ is an inner derivation for all $k$ so that $\mathfrak{m}_{0}(p)$ admits the structure of a restricted Lie algebra (see [10]). To define a $[p]$-operator on $\mathfrak{m}_{0}(p)$, we choose for each $1 \leq k \leq p$, an element $e_{k}^{[p]}$ such that

$$
\operatorname{ad} e_{k}^{[p]}=\left(\operatorname{ad} e_{k}\right)^{p}=0 .
$$

That is, we must choose $e_{k}^{[p]}$ in the center $\mathbb{F} e_{p}$ of the algebra $\mathfrak{m}_{0}(p)$. If we let $\lambda=\left(\lambda_{1}, \ldots, \lambda_{p}\right) \in \mathbb{F}^{p}$, then setting $e_{k}^{[p]}=\lambda_{k} e_{p}$ for each $k$ defines a restricted Lie algebra that we denote by $\mathfrak{m}_{0}^{\lambda}(p)$. Because $p$-fold brackets are zero, if $[p]$ is any restricted Lie algebra operator on $\mathfrak{m}_{0}(p)$, then for all $g, h \in \mathfrak{m}_{0}(p)$, $\alpha \in \mathbb{F}$,

$$
(g+h)^{[p]}=g^{[p]}+h^{[p]} \text { and }(\alpha g)^{[p]}=\alpha^{p} g^{[p]} .
$$

It follows that if $\lambda \in \mathbb{F}^{p}$, then for all $g=\sum \alpha_{k} e_{k} \in \mathfrak{m}_{0}^{\lambda}(p)$,

$$
g^{[p]}=\left(\sum_{k=1}^{p} \alpha_{k}^{p} \lambda_{k}\right) e_{p} .
$$

Everywhere below, we write $\mathfrak{m}_{0}^{\lambda}(p)$ to denote both the Lie algebra $\mathfrak{m}_{0}(p)$ and the restricted Lie algebra $\mathfrak{m}_{0}^{\lambda}(p)$ for a given $\lambda \in \mathbb{F}^{p}$.

A natural question arises: For which $\lambda, \lambda^{\prime} \in \mathbb{F}^{p}$ are the graded restricted Lie algebras $\mathfrak{m}_{0}^{\lambda}(p)$ and $\mathfrak{m}_{0}^{\lambda^{\prime}}(p)$ isomorphic? 
Proposition 2.1. If $\lambda, \lambda^{\prime} \in \mathbb{F}^{p}$, the graded restricted Lie algebras $\mathfrak{m}_{0}^{\lambda}(p)$ and $\mathfrak{m}_{0}^{\lambda^{\prime}}(p)$ are isomorphic if and only if $\lambda_{1}=\mu_{1} \lambda_{1}^{\prime}$ and $\lambda_{2}=\mu_{2} \lambda_{2}^{\prime}$ where $\mu_{1}, \mu_{2} \in \mathbb{F}$ are independent parameters, and

$$
\lambda_{k}=\mu_{2}^{p-1} \mu_{1}^{p(k-3)+2} \lambda_{k}^{\prime},
$$

for $k \in 3, \ldots, p$.

Proof. Assume that there exists a graded restricted Lie algebra isomorphism $\varphi: \mathfrak{m}_{0}^{\lambda}(p) \rightarrow \mathfrak{m}_{0}^{\lambda^{\prime}}(p)$, and let $\varphi\left(e_{1}\right)=\mu_{1} e_{1}, \varphi\left(e_{2}\right)=\mu_{2} e_{2}$ for some $\mu_{1}, \mu_{2} \in \mathbb{F}$. Since $\varphi$ preserves the Lie bracket, we must have $\varphi\left(e_{k}\right)=\mu_{2} \mu_{1}^{k-2} e_{k}, k=$ $3, \ldots, p$. Let $\mu_{k}=\mu_{2} \mu_{1}^{k-2}$ for $3 \leq k \leq p$ so that $\varphi\left(e_{k}\right)=\mu_{k} e_{k}$ for all $k$. Moreover, $\varphi$ preserves the restricted $[p]$-structure so that

$$
\varphi\left(e_{k}^{[p]}\right)=\varphi\left(e_{k}\right)^{[p]^{\prime}}
$$

for all $k$ (here $[p]^{\prime}$ denotes the restricted $[p]$-structure on $\mathfrak{m}_{0}^{\lambda^{\prime}}(p)$ ). Now,

$$
\varphi\left(e_{k}^{[p]}\right)=\varphi\left(\lambda_{k} e_{p}\right)=\lambda_{k} \mu_{p} e_{p} \text { and } \varphi\left(e_{k}\right)^{[p]^{\prime}}=\left(\mu_{k} e_{k}\right)^{[p]^{\prime}}=\mu_{k}^{p} \lambda_{k}^{\prime} e_{p}
$$

so that $\lambda_{k} \mu_{p}=\mu_{k}^{p} \lambda_{k}^{\prime}$, and hence $\lambda_{k}=\mu_{k}^{p} \mu_{p}^{-1} \lambda_{k}^{\prime}$. But $\mu_{k}=\mu_{2} \mu_{1}^{k-2}$, so that

$$
\lambda_{k}=\mu_{2}^{p} \mu_{1}^{p(k-2)} \mu_{2}^{-1} \mu_{1}^{2-p} \lambda_{k}^{\prime}=\mu_{2}^{p-1} \mu_{1}^{p(k-3)+2} \lambda_{k}^{\prime} .
$$

It remains to show that the above condition on the $\lambda_{k}$ gives rise to a graded restricted Lie algebra isomorphism between $\mathfrak{m}_{0}^{\lambda}(p)$ and $\mathfrak{m}_{0}^{\lambda^{\prime}}(p)$. If, for $\mu_{1}, \mu_{2} \in$ $\mathbb{F}$, we define $\varphi\left(e_{1}\right)=\mu_{1} e_{1}, \varphi\left(e_{2}\right)=\mu_{2} e_{2}$ and $\varphi\left(e_{k}\right)=\mu_{2} \mu_{1}^{k-2} e_{k}(3 \leq k \leq p)$, it is easy to check the argument above is reversible, and we obtain a graded isomorphism between the restricted Lie algebras.

\subsection{Cochain Complexes with Trivial Coefficients}

\subsubsection{Ordinary Cochain Complex}

For ordinary Lie algebra cohomology with trivial coefficients, the relevant cochain spaces (with bases) are:

$$
\begin{array}{lrl}
C^{0}\left(\mathfrak{m}_{0}^{\lambda}(p)\right) & =\mathbb{F}, \\
C^{1}\left(\mathfrak{m}_{0}^{\lambda}(p)\right) & =\mathfrak{m}_{0}^{\lambda}(p)^{*}, & \{1\} ; \\
C^{2}\left(\mathfrak{m}_{0}^{\lambda}(p)\right)=\left(\wedge^{2} \mathfrak{m}_{0}^{\lambda}(p)\right)^{*}, & \left.\left\{e^{k} \mid 1 \leq k \leq p\right\} ; 1 \leq i<j \leq p\right\} ; \\
C^{3}\left(\mathfrak{m}_{0}^{\lambda}(p)\right)=\left(\wedge^{3} \mathfrak{m}_{0}^{\lambda}(p)\right)^{*}, & \left\{e^{s, t, u} \mid 1 \leq s<t<u \leq p\right\},
\end{array}
$$


and the differentials are defined by:

$$
\begin{array}{ll}
d^{0}: C^{0}\left(\mathfrak{m}_{0}^{\lambda}(p)\right) \rightarrow C^{1}\left(\mathfrak{m}_{0}^{\lambda}(p)\right) & d^{0}=0 \\
d^{1}: C^{1}\left(\mathfrak{m}_{0}^{\lambda}(p)\right) \rightarrow C^{2}\left(\mathfrak{m}_{0}^{\lambda}(p)\right) & d^{1}(\psi)(g, h)=\psi([g, h]) ; \\
d^{2}: C^{2}\left(\mathfrak{m}_{0}^{\lambda}(p)\right) \rightarrow C^{3}\left(\mathfrak{m}_{0}^{\lambda}(p)\right) & d^{2}(\varphi)(g, h, f)=\varphi([g, h] \wedge f)-\varphi([g, f] \wedge h)+\varphi([h, f] \wedge g) .
\end{array}
$$

The cochain spaces $C^{n}\left(\mathfrak{m}_{0}^{\lambda}(p)\right)$ are graded:

$$
\begin{array}{rr}
C_{k}^{1}\left(\mathfrak{m}_{0}^{\lambda}(p)\right)=\operatorname{span}\left(\left\{e^{k}\right\}\right), & 1 \leq k \leq p ; \\
C_{k}^{2}\left(\mathfrak{m}_{0}^{\lambda}(p)\right)=\operatorname{span}\left(\left\{e^{i, j}\right\}\right), & 1 \leq i<j \leq p, i+j=k, 3 \leq k \leq 2 p-1 ; \\
C_{k}^{3}\left(\mathfrak{m}_{0}^{\lambda}(p)\right)=\operatorname{span}\left(\left\{e^{s, t, u}\right\}\right), & 1 \leq s<t<u \leq p, s+t+u=k, 6 \leq k \leq 3 p-3,
\end{array}
$$

and the differentials are graded maps. For $1 \leq k \leq p$, if we write

$$
d^{1}\left(e^{k}\right)=\sum_{1 \leq i<j \leq p} A_{i j}^{k} e^{i, j},
$$

then for $1 \leq q<r \leq p$, we have

$$
A_{q r}^{k}=d^{1}\left(e^{k}\right)\left(e_{q r}\right)=e^{k}\left[e_{q}, e_{r}\right]= \begin{cases}0 & k=1,2 ; \\ 1 & q=1, r=k-1, k \geq 3 \\ 0 & \text { otherwise. }\end{cases}
$$

Therefore $d^{1}\left(e^{1}\right)=d^{1}\left(e^{2}\right)=0$ and for $3 \leq k \leq p$,

$$
d^{1}\left(e^{k}\right)=e^{1, k-1} .
$$

For $1 \leq i<j \leq p$, if we write

$$
d^{2}\left(e^{i, j}\right)=\sum_{1 \leq s<t<u \leq p} A_{s t u}^{i j} e^{s, t, u} .
$$

Then for $1 \leq l<m<n \leq p$, we have

$$
\begin{aligned}
A_{l m n}^{i j} & =d^{2}\left(e^{i, j}\right)\left(e_{l m n}\right) \\
& =e^{i, j}\left(\left[e_{l}, e_{m}\right] \wedge e_{n}\right)-e^{i, j}\left(\left[e_{l}, e_{n}\right] \wedge e_{m}\right)+e^{i, j}\left(\left[e_{m}, e_{n}\right] \wedge e_{l}\right) .
\end{aligned}
$$

Therefore (44) shows $d^{2}\left(e^{1, j}\right)=0$ for $2 \leq j \leq p$, and for $i \geq 2$,

$$
d^{2}\left(e^{i, j}\right)=e^{1, i-1, j}+e^{1, i, j-i} .
$$




\subsubsection{Restricted Cochain Complex}

For convenience, we include a brief description of the (partial) restricted cochain complex employed below to compute the restricted Lie algebra cohomology with trivial coefficients. We refer the reader to [5] or [4] for a detailed description of this (partial) complex. The first two restricted cochain spaces coincide with the ordinary cochain spaces:

$$
\begin{aligned}
& C_{*}^{0}\left(\mathfrak{m}_{0}^{\lambda}(p)\right)=C^{0}\left(\mathfrak{m}_{0}^{\lambda}(p)\right)=\mathbb{F} \\
& C_{*}^{1}\left(\mathfrak{m}_{0}^{\lambda}(p)\right)=C^{1}\left(\mathfrak{m}_{0}^{\lambda}(p)\right)=\mathfrak{m}_{0}^{\lambda}(p)^{*} .
\end{aligned}
$$

Using the same notation as in [16], we let $\operatorname{Hom}_{\mathrm{Fr}}(V, W)$ denote the set of Frobenius homomorphisms from the $\mathbb{F}$-vector space $V$ to the $\mathbb{F}$-vector space $W$. That is

$$
\operatorname{Hom}_{F r}(V, W)=\left\{f: V \rightarrow W \mid f(\alpha x+\beta y)=\alpha^{p} f(x)+\beta^{p} f(y)\right\}
$$

for all $\alpha, \beta \in \mathbb{F}$ and $x, y \in V$.

If $\varphi \in C^{2}\left(\mathfrak{m}_{0}^{\lambda}(p)\right)$ and $\omega \in \operatorname{Hom}_{\mathrm{Fr}}\left(\mathfrak{m}_{0}^{\lambda}(p), \mathbb{F}\right)$, then we say $\omega$ has the $*-$ property with respect to $\varphi$ if for all $g, h \in \mathfrak{m}_{0}^{\lambda}(p)$ we have

$$
\omega(g+h)=\omega(g)+\omega(h)+\sum_{\substack{g_{i}=g \text { or } h \\ g_{1}=g, g_{2}=h}} \frac{1}{\#(g)} \varphi\left(\left[g_{1}, g_{2}, \ldots, g_{p-1}\right] \wedge g_{p}\right) .
$$

Here $\#(g)$ is the number of factors $g_{i}$ equal to $g$. We remark that $\omega$ has the $*$-property with respect to $\varphi=0$ precisely when $\omega \in \operatorname{Hom}_{\mathrm{Fr}}\left(\mathfrak{m}_{0}^{\lambda}(p), \mathbb{F}\right)$. Moreover, given $\varphi$, we can assign the values of $\omega$ arbitrarily on a basis for $\mathfrak{m}_{0}^{\lambda}(p)$ and use (6) to define $\omega \in \operatorname{Hom}_{\mathrm{Fr}}\left(\mathfrak{m}_{0}^{\lambda}(p), \mathbb{F}\right)$ that has the $*$-property with respect to $\varphi$. We then define the space of restricted 2-cochains as

$$
\begin{array}{r}
C_{*}^{2}\left(\mathfrak{m}_{0}^{\lambda}(p)\right)=\left\{(\varphi, \omega) \mid \varphi \in C^{2}\left(\mathfrak{m}_{0}^{\lambda}(p)\right), \omega \in \operatorname{Hom}_{\mathrm{Fr}}\left(\mathfrak{m}_{0}^{\lambda}(p), F\right)\right. \\
\text { has the } * \text {-property with respect to } \varphi\} .
\end{array}
$$

If $\alpha \in C^{3}\left(\mathfrak{m}_{0}^{\lambda}(p)\right)$ and $\beta \in \mathfrak{m}_{0}^{\lambda}(p)^{*} \otimes \operatorname{Hom}_{\mathrm{Fr}}\left(\mathfrak{m}_{0}^{\lambda}(p), \mathbb{F}\right)$, we say that $\beta$ has the $* *$-property with respect to $\alpha$, if for all $g, h_{1}, h_{2} \in \mathfrak{m}_{0}^{\lambda}(p)$

$$
\begin{aligned}
\beta\left(g, h_{1}+h_{2}\right)= & \beta\left(g, h_{1}\right)+\beta\left(g, h_{2}\right)- \\
& \sum_{\substack{l_{1}, \ldots, l_{p}=1 \mathrm{or} 2 \\
l_{1}=1, l_{2}=2}} \frac{1}{\#\left\{i_{i}=1\right\}} \alpha\left(g \wedge\left[h_{l_{1}}, \cdots, h_{l_{p-1}}\right] \wedge h_{l_{p}}\right) .
\end{aligned}
$$


Again we remark that $\beta$ has the $* *$-property with respect to $\alpha=0$ precisely when $\beta \in \mathfrak{m}_{0}^{\lambda}(p)^{*} \otimes \operatorname{Hom}_{\mathrm{Fr}}\left(\mathfrak{m}_{0}^{\lambda}(p), \mathbb{F}\right)$. Given $\alpha$, we can define the values of $\beta$ arbitrarily on a basis and use (7) to define $\beta \in \mathfrak{m}_{0}^{\lambda}(p)^{*} \otimes \operatorname{Hom}_{\mathrm{Fr}}\left(\mathfrak{m}_{0}^{\lambda}(p), \mathbb{F}\right)$ that has the $* *$-property with respect to $\alpha$. We then define the space of restricted 3-cochains by

$$
\begin{gathered}
C_{*}^{3}\left(\mathfrak{m}_{0}^{\lambda}(p)\right)=\left\{(\alpha, \beta) \mid \alpha \in C^{3}\left(\mathfrak{m}_{0}^{\lambda}(p)\right), \beta \in \mathfrak{m}_{0}^{\lambda}(p)^{*} \otimes \operatorname{Hom}_{\mathrm{Fr}}\left(\mathfrak{m}_{0}^{\lambda}(p), \mathbb{F}\right)\right. \\
\text { has the } * * \text {-property w.r.t. } \alpha\} .
\end{gathered}
$$

We will use the following bases for the restricted cochains:

$$
\begin{array}{rr}
C_{*}^{0}\left(\mathfrak{m}_{0}^{\lambda}(p)\right) & \{1\} ; \\
C_{*}^{1}\left(\mathfrak{m}_{0}^{\lambda}(p)\right) & \left\{e^{k} \mid 1 \leq k \leq p\right\} ; \\
C_{*}^{2}\left(\mathfrak{m}_{0}^{\lambda}(p)\right) & \left\{\left(e^{i, j}, \tilde{e}^{i, j}\right) \mid 1 \leq i<j \leq p\right\} \cup\left\{\left(0, \bar{e}^{k}\right) \mid 1 \leq k \leq p\right\} ;
\end{array}
$$

where $\bar{e}^{k}: \mathfrak{m}_{0}^{\lambda}(p) \rightarrow \mathbb{F}$ is defined by

$$
\bar{e}^{k}\left(\sum_{i=1}^{p} \alpha_{i} e_{i}\right)=\alpha_{k}^{p},
$$

and $\tilde{e}^{i, j}$ is the map $\tilde{e}^{i, j}: \mathfrak{m}_{0}^{\lambda}(p) \rightarrow \mathbb{F}$ that vanishes on the basis and has the *-property with respect to $e^{i, j}$. More generally, given $\varphi \in C^{2}\left(\mathfrak{m}_{0}^{\lambda}(p)\right)$, we let $\tilde{\varphi}: \mathfrak{m}_{0}^{\lambda}(p) \rightarrow \mathbb{F}$ be the map that vanishes on the basis for $\mathfrak{m}_{0}^{\lambda}(p)$ and has the $*$-property with respect to $\varphi$. The restricted differentials are defined by

$$
\begin{array}{ll}
d_{*}^{0}: C_{*}^{0}\left(\mathfrak{m}_{0}^{\lambda}(p)\right) \rightarrow C_{*}^{1}\left(\mathfrak{m}_{0}^{\lambda}(p)\right) & d_{*}^{0}=0 \\
d_{*}^{1}: C_{*}^{1}\left(\mathfrak{m}_{0}^{\lambda}(p)\right) \rightarrow C_{*}^{2}\left(\mathfrak{m}_{0}^{\lambda}(p)\right) & d_{*}^{1}(\psi)=\left(d^{1}(\psi), \operatorname{ind}^{1}(\psi)\right) \\
d_{*}^{2}: C_{*}^{2}\left(\mathfrak{m}_{0}^{\lambda}(p)\right) \rightarrow C_{*}^{3}\left(\mathfrak{m}_{0}^{\lambda}(p)\right) & d_{*}^{2}(\varphi, \omega)=\left(d^{2}(\varphi), \operatorname{ind}^{2}(\varphi, \omega)\right)
\end{array}
$$

where $\operatorname{ind}^{1}(\psi)(g):=\psi\left(g^{[p]}\right)$ and $\operatorname{ind}^{2}(\varphi, \omega)(g, h):=\varphi\left(g \wedge h^{[p]}\right)$.

If $\psi \in C_{*}^{1}\left(\mathfrak{m}_{0}^{\lambda}(p)\right)$ and $(\varphi, \omega) \in C_{*}^{2}\left(\mathfrak{m}_{0}^{\lambda}(p)\right)$, then $\operatorname{ind}^{1}(\psi)$ has the *property with respect to $d^{1}(\psi)$ and $\operatorname{ind}^{2}(\varphi, \omega)$ has the $* *$-property with respect to $d^{2}(\varphi)$ [5]. If $g=\sum \alpha_{i} e_{i}, h=\sum \beta_{i} e_{i}, \psi=\sum \mu_{i} e^{i}$ and $\varphi=\sum \sigma_{i j} e^{i, j}$, then

$$
\operatorname{ind}^{1}(\psi)(g)=\mu_{p}\left(\sum_{j=1}^{p} \alpha_{j}^{p} \lambda_{j}\right)
$$


and

$$
\operatorname{ind}^{2}(\varphi, \omega)(g, h)=\left(\sum_{i=1}^{p} \beta_{i}^{p} \lambda_{i}\right)\left(\sum_{j=1}^{p-1} \alpha_{j} \sigma_{j p}\right)
$$

Remark. For a given $\varphi \in C^{2}\left(\mathfrak{m}_{0}^{\lambda}(p)\right)$, if $(\varphi, \omega),\left(\varphi, \omega^{\prime}\right) \in C_{*}^{2}\left(\mathfrak{m}_{0}^{\lambda}(p)\right)$, then $d_{*}^{2}(\varphi, \omega)=d_{*}^{2}\left(\varphi, \omega^{\prime}\right)$. In particular, with trivial coefficients, $\operatorname{ind}^{2}(\varphi, \omega)$ depends only on $\varphi$.

\section{The Cohomology $H^{1}\left(\mathfrak{m}_{0}^{\lambda}(p)\right)$ and $H_{*}^{1}\left(\mathfrak{m}_{0}^{\lambda}(p)\right)$}

Theorem 3.1. If $p \geq 3$ and $\lambda \in \mathbb{F}^{p}$, then

$$
H^{1}\left(\mathfrak{m}_{0}^{\lambda}(p)\right)=H_{*}^{1}\left(\mathfrak{m}_{0}^{\lambda}(p)\right)
$$

and the classes of $\left\{e^{1}, e^{2}\right\}$ form a basis.

Proof. It follows easily from (3) that $\operatorname{dim}\left(\operatorname{ker} d^{1}\right)=2$ and $\left\{e^{1}, e^{2}\right\}$ is a basis for this kernel. Moreover $d^{0}=0$, so that

$$
H^{1}\left(\mathfrak{m}_{0}^{\lambda}(p)\right) \cong \operatorname{ker} d^{1}=\mathbb{F} e^{1} \oplus \mathbb{F} e^{2} .
$$

Now, $H_{*}^{1}\left(\mathfrak{m}_{0}^{\lambda}(p)\right)$ consists of those ordinary cohomology classes $[\psi] \in$ $H^{1}\left(\mathfrak{m}_{0}^{\lambda}(p)\right)$ for which $\operatorname{ind}^{1}(\psi)=0$ [5]. If $\psi=\sum_{k=1}^{p} \mu_{k} e^{k}$ is any ordinary cocycle, then $\mu_{p}=0(p \geq 3)$ so that for any $g \in \mathfrak{m}_{0}^{\lambda}(p)$, we have

$$
\operatorname{ind}^{1}(\psi)(g)=\psi\left(g^{[p]}\right)=\mu_{p}\left(\sum_{k=1}^{p} \alpha_{k}^{p} \lambda_{k}\right)=0
$$

and hence $H_{*}^{1}\left(\mathfrak{m}_{0}^{\lambda}(p)\right)=H^{1}\left(\mathfrak{m}_{0}(p)\right)$.

Remark. For $p \geq 3$, formula (3) shows that for $3 \leq k \leq p, d^{1}\left(e_{k}\right)=e^{1, k-1}$, so the set $\left\{e^{1,2}, \ldots, e^{1, p-1}\right\}$ is a basis for the image $d^{1}\left(C^{1}\left(\mathfrak{m}_{0}^{\lambda}(p)\right)\right.$.

The above calculation of the ordinary cohomology $H^{1}\left(\mathfrak{m}_{0}^{\lambda}(p)\right)$ is valid also in the case $p=2$, but the restricted cohomology depends on $\lambda$ in this case. In particular, if $p=2$, and $\lambda=(0,0)$, then $\operatorname{ind}^{1}(\psi)(g)=0$ for all $g \in \mathfrak{m}_{0}$ and hence $H_{*}^{1}\left(\mathfrak{m}_{0}^{\lambda}(2)\right)=H^{1}\left(\mathfrak{m}_{0}^{\lambda}(2)\right)$. If $\lambda \neq(0,0)$, then $\operatorname{ind}^{1}(\psi)(g)=0$ for all $g \in \mathfrak{m}_{0}$ if and only if $\mu_{2}=0$ so that $\operatorname{ker} d_{*}^{1}=\left\{e^{1}\right\}$ and $H_{*}^{1}\left(\mathfrak{m}_{0}^{\lambda}(2)\right)$ is one dimensional. 


\section{The Cohomology $H^{2}\left(\mathfrak{m}_{0}^{\lambda}(p)\right)$ and $H_{*}^{2}\left(\mathfrak{m}_{0}^{\lambda}(p)\right)$}

\subsection{Ordinary Cohomology}

Theorem 4.1. If $p=2, H^{2}\left(\mathfrak{m}_{0}^{\lambda}(2)\right) \cong C^{2}\left(\mathfrak{m}_{0}^{\lambda}(2)\right)=\operatorname{span}\left(\left\{e^{1,2}\right\}\right)$ is 1dimensional.

If $p \geq 3$, then

$$
\operatorname{dim}\left(H^{2}\left(\mathfrak{m}_{0}^{\lambda}(p)\right)\right)=\frac{p+1}{2}
$$

and the cohomology classes of the cocycles $\left\{e^{1, p}, \varphi_{5}, \varphi_{7}, \varphi_{9}, \ldots, \varphi_{p+2}\right\}$ form a basis, where

$$
\varphi_{k}=e^{2, k-2}-e^{3, k-3}+\cdots+(-1)^{\left\lfloor\frac{k}{2}\right\rfloor} e^{\left\lfloor\frac{k}{2}\right\rfloor, k-\left\lfloor\frac{k}{2}\right\rfloor} .
$$

Proof. If $p=2$, the algebra $\mathfrak{m}_{0}^{\lambda}(2)$ is abelian so that $d^{1}=d^{2}=0$.

If $p \geq 3$, the proof of Theorem 3.1 and the remark following the proof show that we have $\operatorname{dim}\left(\operatorname{im} d^{1}\right)=p-2$ and $\left\{e^{12}, \ldots, e^{1 p-1}\right\}$ is a basis for this image. If $p=3, d^{2}=0$ so that the ordinary cohomology $H^{2}\left(\mathfrak{m}_{0}^{\lambda}(3)\right)$ is 2 -dimensional and has a basis consisting of the classes of the cocyles $e^{13}$ and $\varphi_{5}=e^{23}$.

If $p>3$, a basis for ker $d^{2}$ is

$$
B\left(Z^{2}\right)=\left\{e^{1,2}, e^{1,3}, \ldots, e^{1, p}, \varphi_{5}, \varphi_{7}, \ldots, \varphi_{p+2}\right\}
$$

where $\varphi_{k}=e^{2, k-2}-e^{3, k-3}+\cdots+(-1)^{\left\lfloor\frac{k}{2}\right\rfloor} e^{\left\lfloor\frac{k}{2}\right\rfloor, k-\left\lfloor\frac{k}{2}\right\rfloor}$. To see this, note that in order to have $d^{2}=0$, by using formula (5), it is clear that any cocycle element has to include either the basis element $e^{1, k}$, and in this case this is a cocycle element, or it has to have one and only one element of type $e^{2, k}$ in the combination, and all those are listed above. The linear independence of the listed cocycle elements are clear.

Example. If $p=7$, then a basis for ker $d^{2}$ is

$$
B\left(Z^{2}\right)=\left\{e^{1,2}, e^{1,3}, e^{1,4}, e^{1,5}, e^{1,6}, e^{1,7}, e^{2,3}, e^{2,5}-e^{3,4}, e^{2,7}-e^{3,6}+e^{4,5}\right\} .
$$

The cohomology $H^{2}\left(\mathfrak{m}_{0}^{\lambda}(7)\right)$ has a basis consisting of the classes of

$$
\left\{e^{1,7}, e^{2,3}, e^{2,5}-e^{3,4}, e^{2,7}-e^{3,6}+e^{4,5}\right\} .
$$




\subsection{Restricted Cohomology with $\lambda=0$}

If $\lambda=0$, then (9) shows that ind $^{2}=0$ so that every ordinary 2-cocycle $\varphi \in$ $C^{2}\left(\mathfrak{m}_{0}^{0}(p)\right)$ gives rise to a restricted 2-cocycle $(\varphi, \tilde{\varphi}) \in C_{*}^{2}\left(\mathfrak{m}_{0}^{0}(p)\right)$. Therefore we can construct a basis for $H_{*}^{2}\left(\mathfrak{m}_{0}^{0}(p)\right)$ from a basis for $H^{2}\left(\mathfrak{m}_{0}^{0}(p)\right)$ and the restricted 2-cocycles $\left(0, \bar{e}_{i}\right), 1 \leq i \leq p$. We summarize this in the following theorem. As before, the case $p=2$ is treated separately.

Theorem 4.2. Let $\lambda=0$. If $p=2$, then

$$
H_{*}^{2}\left(\mathfrak{m}_{0}^{0}(2)\right) \cong C_{*}^{2}\left(\mathfrak{m}_{0}^{0}(2)\right)=\operatorname{span}\left(\left\{\left(0, \bar{e}^{1}\right),\left(0, \bar{e}^{2}\right),\left(e^{1,2}, \tilde{e}^{1,2}\right)\right\}\right)
$$

is 3-dimensional. If $p \geq 3$, then

$$
\operatorname{dim}\left(H_{*}^{2}\left(\mathfrak{m}_{0}^{0}(p)\right)\right)=\frac{3 p+1}{2}
$$

and the cohomology classes of

$$
\left\{\left(0, \bar{e}^{1}\right), \ldots,\left(0, \bar{e}^{p}\right),\left(e^{1, p}, \tilde{e}^{1, p}\right),\left(\varphi_{5}, \tilde{\varphi}_{5}\right),\left(\varphi_{7}, \tilde{\varphi}_{7}\right), \ldots,\left(\varphi_{p+2}, \tilde{\varphi}_{p+2}\right)\right\}
$$

form a basis.

Proof. If $p=2$, the algebra $\mathfrak{m}_{0}^{0}(2)$ is abelian, and $\operatorname{ind}^{1}=\operatorname{ind}^{2}=0$ so that $d_{*}^{1}=d_{*}^{2}=0$.

If $p \geq 3$, the proof of Theorem 4.1 shows that

$$
\left\{e^{1,2}, \ldots, e^{1, p}, \varphi_{5}, \varphi_{7}, \ldots, \varphi_{p+2}\right\}
$$

is a basis for the kernel of $d^{2}$. Since $\lambda=0$, (9) implies ind ${ }^{2}=0$ so that

$$
\left\{\left(e^{1,2}, \tilde{e}^{1,2}\right), \ldots,\left(e^{1, p}, \tilde{e}^{1, p}\right),\left(\varphi_{5}, \tilde{\varphi}_{5}\right),\left(\varphi_{7}, \tilde{\varphi}_{7}\right), \ldots,\left(\varphi_{p+2}, \tilde{\varphi}_{p+2}\right)\right\}
$$

is a linearly independent subset of ker $d_{*}^{2}$. Moreover, for $1 \leq k \leq p$, the maps $\left(0, \bar{e}^{k}\right)$ are also in the kernel of $d_{*}^{2}$ and the set

$B\left(Z_{*}^{2}\right)=\left\{\left(0, \bar{e}^{1}\right), \ldots,\left(0, \bar{e}^{p}\right),\left(e^{1,2}, \tilde{e}^{1,2}\right), \ldots,\left(e^{1, p}, \tilde{e}^{1, p}\right),\left(\varphi_{5}, \tilde{\varphi}_{5}\right), \ldots,\left(\varphi_{p+2}, \tilde{\varphi}_{p+2}\right)\right\}$

is a basis $B\left(Z_{*}^{2}\right)$ for ker $d_{*}^{2}$. We can redefine $\tilde{e}^{1, k-1}=\operatorname{ind}^{1}\left(e^{k}\right)$ for $3 \leq k \leq p$ without affecting the coboundary (see the remark at the end of Section 3) so that $d_{*}^{1}\left(e^{k}\right)=\left(e^{1, k-1}, \tilde{e}^{1, k-1}\right)$, and the set

$$
\left\{d_{*}^{1}\left(e^{k}\right) \mid 3 \leq k \leq p\right\} \subseteq B\left(Z_{*}^{2}\right)
$$


forms a basis for the image $d_{*}^{1}\left(C^{1}\left(\mathfrak{m}_{0}^{0}(p)\right)\right)$. It follows that

$$
\operatorname{dim}\left(\operatorname{ker} d_{*}^{2}\right)=p+\frac{3(p-1)}{2}=\frac{5 p-3}{2}
$$

and $\operatorname{dim}\left(\operatorname{im} d_{*}^{1}\right)=p-2$ which completes the proof.

Remark. Another approach for determining the dimensions in Theorem 4.2 for $p \geq 2$ uses the six-term exact sequence in [9]:

$$
\begin{aligned}
& 0 \longrightarrow H_{*}^{1}(\mathfrak{g}, M) \longrightarrow H^{1}(\mathfrak{g}, M) \longrightarrow \operatorname{Hom}_{\mathrm{Fr}}\left(\mathfrak{g}, M^{\mathfrak{g}}\right) \longrightarrow \\
& \longrightarrow H_{*}^{2}(\mathfrak{g}, M) \longrightarrow H^{2}(\mathfrak{g}, M) \longrightarrow \operatorname{Hom}_{\mathrm{Fr}}\left(\mathfrak{g}, H^{1}(\mathfrak{g}, M)\right)
\end{aligned}
$$

If $\mathfrak{g}=\mathfrak{m}_{0}^{\lambda}(p)$ and $M=\mathbb{F}$, the map $\Delta: H^{2}(\mathfrak{g}, M) \rightarrow \operatorname{Hom}_{\mathrm{Fr}}\left(\mathfrak{g}, H^{1}(\mathfrak{g}, M)\right)$ in (10) is given by

$$
\Delta_{\varphi}(g) \cdot h=\varphi\left(g, h^{[p]}\right)=\operatorname{ind}^{2}(\varphi, \omega)(g, h)
$$

where $\varphi \in C^{2}(\mathfrak{g})$ and $g, h \in \mathfrak{g}($ see [16] $)$. If $p \geq 3$, the map $H_{*}^{1}\left(\mathfrak{m}_{0}^{\lambda}(p)\right) \rightarrow$ $H^{1}\left(\mathfrak{m}_{0}^{\lambda}(p)\right)$ is an isomorphism so that the sequence (10) decouples and the sequence

$$
0 \longrightarrow \operatorname{Hom}_{\mathrm{Fr}}\left(\mathfrak{m}_{0}^{\lambda}, \mathbb{F}\right) \longrightarrow H_{*}^{2}\left(\mathfrak{m}_{0}^{\lambda}\right) \longrightarrow H^{2}\left(\mathfrak{m}_{0}^{\lambda}\right) \stackrel{\Delta}{\longrightarrow} \operatorname{Hom}_{\mathrm{Fr}}\left(\mathfrak{m}_{0}^{\lambda}, H^{1}\left(\mathfrak{m}_{0}^{\lambda}\right)\right)
$$

is exact. If $\lambda=0, \Delta=0$ so that the sequence (11) reduces to the short exact sequence

$$
0 \longrightarrow \operatorname{Hom}_{\mathrm{Fr}}\left(\mathfrak{m}_{0}^{\lambda}, \mathbb{F}\right) \longrightarrow H_{*}^{2}\left(\mathfrak{m}_{0}^{\lambda}\right) \longrightarrow H^{2}\left(\mathfrak{m}_{0}^{\lambda}\right) \longrightarrow
$$

\subsection{Restricted Cohomology with $\lambda \neq 0$}

If $\varphi=\sum \sigma_{i j} e^{i, j}$ and $(\varphi, \omega) \in C_{*}^{2}\left(\mathfrak{m}_{0}^{\lambda}\right)$, then (9) shows that

$$
\operatorname{ind}^{2}(\varphi, \omega)\left(e_{j}, e_{i}\right)=\lambda_{i} \sigma_{j p} .
$$

Therefore, if $\lambda \neq 0$, then $d_{*}^{2}(\varphi, \omega)=\left(d^{2} \varphi\right.$, ind $\left.{ }^{2}(\varphi, \omega)\right)=(0,0)$ if and only if $d^{2} \varphi=0$ and $\sigma_{1 p}=\sigma_{2 p}=\cdots=\sigma_{p-1 p}=0$. This observation, together with the calculation of the basis $B\left(Z_{*}^{2}\right)$ of $d_{*}^{2}$ in the proof of Theorem 4.2, proves the following 
Lemma 4.3. Let $\lambda \neq 0$. If $p=2$, then $\operatorname{ker} d_{*}^{2}=\operatorname{span}\left(\left\{\left(0, \bar{e}^{1}\right),\left(0, \bar{e}^{2}\right)\right\}\right)$. If $p \geq 3$ and then a basis for the $\operatorname{ker} d_{*}^{2}$ is

$$
B\left(Z_{*}^{2}\right)-\left\{\left(e^{1, p}, \tilde{e}^{1, p}\right),\left(\varphi_{p+2}, \tilde{\varphi}_{p+2}\right)\right\} .
$$

As above, we treat the case $p=2$ separately. It is interesting to note that when $\lambda \neq 0$, the basis for the cohomology $H_{*}^{2}\left(\mathfrak{m}_{0}^{\lambda}(2)\right)$ depends on which coordinate of $\lambda$ is non-zero, whereas this is not the case for $p \geq 3$.

Theorem 4.4. If $p=2$ and $\lambda=\left(\lambda_{1}, \lambda_{2}\right) \neq 0$, then $\operatorname{dim}\left(H_{*}^{2}\left(\mathfrak{m}_{0}^{\lambda}(2)\right)\right)=1$.

If $\lambda_{2} \neq 0$, then the cohomology class of the cocycle $\left(0, \bar{e}^{1}\right)$ is a basis for $H_{*}^{2}\left(\mathfrak{m}_{0}^{\lambda}(2)\right)$.

If $\lambda_{2}=0$, then the cohomology class of the cocycle $\left(0, \bar{e}^{2}\right)$ is a basis for $H_{*}^{2}\left(\mathfrak{m}_{0}^{\lambda}(2)\right)$.

Proof. If $p=2$, then the ordinary differentials $d^{1}=d^{2}=0$, and (8) shows that $\operatorname{ind}^{1}\left(e^{1}\right)=0$ and $\operatorname{ind}^{1}\left(e^{2}\right)=\lambda_{1} \bar{e}^{1}+\lambda_{2} \bar{e}^{2}$. It follows that

$$
\left\{\left(0, \lambda_{1} \bar{e}^{1}+\lambda_{2} \bar{e}^{2}\right)\right\}
$$

is a basis for the image $d_{*}^{1}\left(\mathfrak{m}_{0}^{\lambda}(2)\right)$. Moreover, $(\varphi, \omega) \in$ ker $d_{*}^{2}$ if and only if $\operatorname{ind}^{2}(\varphi, \omega)=0$ if and only if $(\varphi, \omega) \in \operatorname{span}\left(\left\{\left(0, \bar{e}^{1}\right),\left(0, \bar{e}^{2}\right)\right\}\right.$ by (9). If $\lambda_{2} \neq 0$, then

$$
\operatorname{ker}\left(d_{*}^{2}\right)=\operatorname{span}\left(\left\{\left(0, \bar{e}^{1}\right),\left(0, \bar{e}^{2}\right)\right\}\right)=\operatorname{span}\left(\left\{\left(0, \bar{e}^{1}\right),\left(0, \lambda_{1} \bar{e}^{-1}+\lambda_{2} \bar{e}^{2}\right)\right\}\right)
$$

so that the cohomology class of $\left(0, \bar{e}^{1}\right)$ is a basis for $H_{*}^{2}\left(\mathfrak{m}_{0}^{\lambda}(2)\right)$. If $\lambda_{2}=0$, then the image

$$
d_{*}^{1}\left(\mathfrak{m}_{0}^{\lambda}(2)\right)=\operatorname{span}\left(\left\{\left(0, \lambda_{1} \bar{e}^{1}\right)\right\}\right)=\operatorname{span}\left(\left\{\left(0, \bar{e}^{1}\right)\right\}\right)
$$

so that that the cohomology class of $\left(0, \bar{e}^{2}\right)$ is a basis for $H_{*}^{2}\left(\mathfrak{m}_{0}^{\lambda}(2)\right)$.

Theorem 4.5. If $p \geq 3$ and $\lambda \neq 0$, then

$$
\operatorname{dim}\left(H_{*}^{2}\left(\mathfrak{m}_{0}^{\lambda}(p)\right)\right)=\frac{3 p-3}{2}
$$

and the cohomology classes of

$$
\left\{\left(0, \bar{e}^{1}\right), \ldots,\left(0, \bar{e}^{p}\right),\left(\varphi_{5}, \tilde{\varphi}_{5}\right),\left(\varphi_{7}, \tilde{\varphi}_{7}\right) \ldots,\left(\varphi_{p}, \tilde{\varphi}_{p}\right)\right\}
$$

form a basis. In particular, the cohomology $H_{*}^{2}\left(\mathfrak{m}_{0}^{\lambda}(3)\right)$ has a basis consisting of the cohomology classes of the cocycles

$$
\left\{\left(0, \bar{e}^{1}\right),\left(0, \bar{e}^{2}\right),\left(0, \bar{e}^{3}\right)\right\} .
$$

Proof. This follows immediately from Lemma 4.3. 


\section{One-dimensional Central Extensions}

It is well known that one-dimensional central extensions of an ordinary Lie algebra $\mathfrak{g}$ are parameterized by the cohomology group $H^{2}(\mathfrak{g})$ [8]. Likewise, restricted one-dimensional central extensions of an ordinary Lie algebra $\mathfrak{g}$ are parameterized by the restricted cohomology group $H_{*}^{2}(\mathfrak{g})[5]$. In the case that $\mathfrak{g}$ is a restricted simple Lie algebra, the authors in [3] use the exact sequence (10) to show that $H_{*}^{2}(\mathfrak{g})=H^{2}(\mathfrak{g}) \oplus \operatorname{Hom}_{F r}(\mathfrak{g}, \mathbb{F})$ and the cohomology classes of the cocyles $\left(0, \bar{e}^{k}\right), 1 \leq k \leq \operatorname{dim} \mathfrak{g}$ span a (dim $\left.\mathfrak{g}\right)$-dimensional subspace of $H^{*}(\mathfrak{g})$. Moreover, if $E_{k}$ denotes the one-dimensional restricted central extension of $\mathfrak{g}$ determined by the cohomology class of the cocycle $\left(0, \bar{e}^{k}\right)$, then $E_{k}=\mathfrak{g} \oplus \mathbb{F} c$ as a $\mathbb{F}$-vector space. For all $1 \leq i, j \leq \operatorname{dim} \mathfrak{g}$,

$$
\begin{aligned}
{\left[x_{i}, x_{j}\right] } & =\left[x_{i}, x_{j}\right]_{\mathfrak{g}} \\
{\left[x_{i}, c\right] } & =0 \\
e_{i}^{[p]} & =x_{i}^{[p]_{\mathfrak{g}}}+\delta_{k, i} c ; \\
c^{[p]} & =0,
\end{aligned}
$$

where $[\cdot, \cdot]_{\mathfrak{g}}$ and $\cdot[p]_{\mathfrak{g}}$ denote the Lie bracket and $[p]$-operation in $\mathfrak{g}$ respectively, and $\delta$ denotes the Kronecker delta-function.

If $\lambda=0$, the restricted Lie algebra $\mathfrak{g}=\mathfrak{m}_{0}^{0}(p)$ is not simple, but the the exact sequence (12) shows that Theorem 3.1 and and Corollary 3.2 in [3] also hold for the algebra $\mathfrak{m}_{0}^{0}(p)$, and we have

Theorem 5.1. If $p \geq 2$, then

$$
H_{*}^{2}\left(\mathfrak{m}_{0}^{0}(p)\right)=H^{2}\left(\mathfrak{m}_{0}^{0}(p)\right) \oplus \operatorname{Hom}_{\mathrm{Fr}}\left(\mathfrak{m}_{0}^{0}(p), \mathbb{F}\right),
$$

and there is a p-dimensional subspace of $H_{*}^{2}\left(\mathfrak{m}_{0}^{0}(p)\right)$ spanned by the cohomology classes of the cocycles $\left(0, \bar{e}^{k}\right)$ such that if $E_{k}$ denotes the corresponding one-dimensional restricted central extension, then $E_{k}=\mathfrak{m}_{0}^{0}(p) \oplus \mathbb{F} c$ and the bracket and $[p]$-operator are given by (13).

If $\lambda \neq 0$, then an ordinary cocycle $\varphi \in C^{2}\left(\mathfrak{m}_{0}^{\lambda}(p)\right)$ need not give rise to a restricted cocycle $(\varphi, \omega) \in C_{*}^{2}\left(\mathfrak{m}_{0}^{\lambda}(p)\right)$. For example, if $p=7$, then $\varphi=e^{1,7}$ is an ordinary cocycle but (9) shows that

$$
\operatorname{ind}^{2}\left(e^{1,7}, \tilde{e}^{1,7}\right)\left(e_{1}, e_{j}\right)=\lambda_{j}
$$

so that $\operatorname{ind}^{2} \neq 0$ if $\lambda_{j} \neq 0$. 
In any case, the sequence (12) shows that $H_{*}^{2}\left(\mathfrak{m}_{0}^{\lambda}(p)\right)$ always has a $p$ dimensional subspace spanned by the cohomology classes of the cocyles $\left(0, \bar{e}^{k}\right)$, $1 \leq k \leq p$. Each of the corresponding restricted one-dimensional central extensions $E_{k}$ of $\mathfrak{m}_{0}^{\lambda}(p)$ are trivial when considered as ordinary one-dimensional central extensions.

\section{References}

[1] A. Caranti, M.F. Mattarei, and M.F. Newman. Graded Lie algebras of maximal class. Trans. Amer. Math. Soc., 39(10):4021-4051, 1997.

[2] A. Caranti and M.F. Newman. Graded Lie algebras of maximal class ii. Journal of Algebra, 229(2):750-784, 2000.

[3] T.J. Evans and A. Fialowski. Restricted one-dimensional central extensions of restricted simple Lie algebras. Linear Algebra and Its Applications, 513C:96-102, 2017.

[4] T.J. Evans, A. Fialowski, and M. Penkava. Restricted cohomology of modular Witt algebras. Proc. Amer. Math. Soc., 144:1877-1886, 2016.

[5] T.J. Evans and D.B. Fuchs. A complex for the cohomology of restricted Lie algebras. Journal of Fixed Point Theory and Applications, 3(1):159179, May 2008.

[6] A. Fialowski. On the classification of graded Lie algebras with two generators. Moscow Univ. Math. Bull., 38(2):76-79, 1983.

[7] A. Fialowski and D. Millionschikov. Cohomology of graded Lie algebras of maximal class. Journal of Algebra, 296(1):157-176, 2006.

[8] D.B. Fuchs. Cohomology of Infinite Dimensional Lie Algebras. Consultants Bureau, 1986.

[9] G. Hochschild. Cohomology of restricted Lie algebras. American Journal of Mathematics, 76(3):555-580, 1954.

[10] N. Jacobson. Lie Algebras. John Wiley, 1962.

[11] G. Jurman. Graded Lie algebras of maximal class iii. Journal of Algebra, 284(2):435-461, 2005. 
[12] D. Millionschikov. Graded filiform Lie algebras and symplectic nilmanifolds. Amer. Math. Soc. Transl., Ser. 2(212):259-279, 2004.

[13] Y. Nikolayevsky and I. Tsartsaflis. Cohomology of n-graded Lie algebras of maximal class over $\mathbb{Z}_{2}$. Journal of Lie Theory, 27(2):529-544, 2017.

[14] I. Tsartsaflis. On the betti numbers of filiform Lie algebras over fields of characteristic two. Revista de la Union Matematica Argentina, 58(1):95106, 2017.

[15] M. Vergne. Cohomologie des algebres de lie nilpotentes. application a l'etude de la variete des algebres de lie nilpotentes. Bull. Sic. Math. France, 98:81-116, 1970.

[16] F. Viviani. Restricted inifinitesimal deformations of restricted simple Lie algebras. Journal of Algebra and Its Applications, 11(5):1250091 (19 pages), 2012. 